\title{
Role of PET/CT in patients with unexplained rising alpha fetoprotein post HCC interventional management
}

\author{
Samar Ramzy Ragheb*, Amr Mahmoud Ahmed Abelsamad, Moustafa Mahmoud Abelkawy and \\ Osama Abu Elnaga Khalaf Nasr
}

\begin{abstract}
Background: Positron emission tomography-computed tomography (PET/CT) is considered a powerful modality in the follow-up of hepatocellular carcinoma (HCC) patients. In this study, PET/CT was done in an evaluation of patients with unexplained rising alpha fetoprotein (AFP) post hepatocellular carcinoma (HCC) interventional management in 40 patients (16 females and 24 males); their age ranged from 25 to 82 years, had undergone interventional management for HCC and underwent PET/CT follow-up within an 8-month duration from their intervention. Whole-body PET/CT was performed after injection of (18)-FDG, and the results were read in a masked manner by two specialists, and diagnostic performance was assessed from the results of consensus masked reading. All the results were evaluated with the Barcelona criteria and biopsy correlation.
\end{abstract}

Results: During the follow-up PET/CT, 24 patients had complete response and 8 patients showed focal residual while the rest 8 patients showed newly developed lesions.

Conclusion: PET/CT is an excellent method for the evaluation of HCC patients with equivocal results after interventional management.

Keywords: Positron emission tomography, Computed tomography, Alpha fetoprotein, Hepatocellular carcinoma

\section{Background}

Hepatocellular carcinoma $(\mathrm{HCC})$ arises in more than $75 \%$ of patients with advanced liver cirrhosis. The responsible factor is known in $90 \%$ of cases. However, the main risk factors for liver cirrhosis and developing $\mathrm{HCC}$ are strongly related to the geographic region [1].

Worldwide, the most important risk factors are viral hepatitis, alcohol, and aflatoxin exposure. Fifty-four percent of all HCCs can be attributed to hepatitis B and $31 \%$ to hepatitis C. Interestingly, signs of advanced cirrhosis such as portal hypertension correlate with the development of HCC [2-4].

The widely accepted imaging modalities for staging HCC are dynamic computed tomography (CT) and contrast-enhanced magnetic resonance imaging (MRI) [5-7]. However, CT and MRI have a limited ability to identify distant metastases. Previous studies

* Correspondence: doc_mina2004@yahoo.com

Department of Radiodiagnosis, Faculty of Medicine, Ain Shams University, Cairo, Egypt

\section{Springer Open}

(c) The Author(s). 2020 Open Access This article is licensed under a Creative Commons Attribution 4.0 International License, which permits use, sharing, adaptation, distribution and reproduction in any medium or format, as long as you give appropriate credit to the original author(s) and the source, provide a link to the Creative Commons licence, and indicate if changes were made. The images or other third party material in this article are included in the article's Creative Commons licence, unless indicated otherwise in a credit line to the material. If material is not included in the article's Creative Commons licence and your intended use is not permitted by statutory regulation or exceeds the permitted use, you will need to obtain permission directly from the copyright holder. To view a copy of this licence, visit http://creativecommons.org/licenses/by/4.0/. 
Table 1 Demographic data of the studied patients $($ no. $=40)$

\begin{tabular}{lll}
\hline Demographic Data & \multicolumn{2}{l}{ All studied patients $(n=40)$} \\
\cline { 2 - 3 } & No. & $\%$ \\
\hline Gender & 14 & $35 \%$ \\
$\quad$ Female & 26 & $65 \%$ \\
$\quad$ Male & & \\
Age & $53 \pm 17$ & \\
Mean \pm SD & $58(23-80)$ & \\
Median (range) &
\end{tabular}

have detected the role of FDG PET/CT in detecting distant metastases in various types of malignancies $[8,9]$.

Using 18F-FDG PET/CT may be promising in the early evaluation of post-therapy effect and the presence of intrahepatic recurrent or extrahepatic metastatic lesions [10, 11]. However, PET/CT usage in hepatocelluar carcinoma (HCC) remains controversial because of concerns about the relatively low sensitivity, especially for detecting a well-differentiated HCC $[12,13]$. Indeed, the diagnostic performance of 18F-FDG PET may strongly depend on biopsy results: one study reported that in patients following transplantation, it detected only $25 \%$ of intrahepatic recurrence cases, but $92.9 \%$ of extrahepatic metastases larger than $1 \mathrm{~cm}$. In addition, 18F-FDG uptake is increased in inflamed tissue, which can contribute to false positive results $[14,15]$.

\section{Methods}

The current study included 40 cases of HCCs were treated with radiofrequency ablation (RFA), microwave ablation (MWA), transarterial chemoembolization (TACE), and combined technique. Follow-up $\mathrm{PET} / \mathrm{CT}$ was done for all cases. It was done during the period from July 2016 till January 2017 and was approved by the local research ethical committee at our University. An informed consent was obtained from all patients.

Table 2 Type of hepatitis of all studied patients

\begin{tabular}{lll}
\hline Type of & \multicolumn{2}{l}{ All studied patients $(n=40)$} \\
\cline { 2 - 3 } hepatitis & No. & $\%$ \\
\hline HBV & 24 & $60 \%$ \\
HCV & 6 & $15 \%$ \\
Alcoholic liver & 10 & $25 \%$ \\
\hline
\end{tabular}

Table 3 Child-Pugh classification of all studied patients

\begin{tabular}{lll}
\hline $\begin{array}{l}\text { Child-Pugh } \\
\text { classification }\end{array}$ & \multicolumn{2}{l}{ All studied patients $(n=40)$} \\
\cline { 2 - 3 } & No. & $\%$ \\
\hline A & 18 & $45 \%$ \\
B & 14 & $35 \%$ \\
C & 8 & $20 \%$ \\
\hline
\end{tabular}

\section{Inclusion criteria}

Any HCC patient, regarding age and sex, was treated with radiofrequency ablation (RFA), microwave ablation (MWA), transarterial chemoembolization (TACE), and combined technique.

\section{Exclusion criteria}

1. Patients with contraindication to contrast: patients with disturbed renal function test (if creatinine $>2$ ), patients with glomerular filtration rate $<30 \mathrm{ml}$ per min per $1.73 \mathrm{~m}^{2}$ or any acute renal insufficiency related to the hepato-renal syndrome or perioperative liver transplantation

2. Patients with metastatic HCC

3. Patients with uncontrolled serum glucose level

4. Patients who are pregnant

\section{Patient preparation}

All patients were asked to fast and rest for a minimum of $6 \mathrm{~h}$ before undergoing the examination. Activities including talking, chewing, and walking were restricted. Serum glucose levels were measured $1 \mathrm{~h}$ before FDG (fluorodeoxyglucose) injection to ensure that the included patients had a level below $150 \mathrm{mg} /$ $\mathrm{dl}$; the examination was postponed if the level was above $150 \mathrm{mg} / \mathrm{dl}$. No oral contrast agent was administered. In addition, all patients were instructed to void preceding the examination. Patients were placed in a lying down position with raised arms.

\section{$\mathrm{PET} / \mathrm{CT}$ technique}

18F-FDG PET/CT study and image analysis PET studies were performed after various procedures with unexplained elevated alpha fetoprotein for all patients using a dedicated PET scanner (DST PET/CT;

Table 4 TNM staging of HCC in all studied patients

\begin{tabular}{lll}
\hline \multirow{2}{*}{ TNM } & \multicolumn{2}{l}{ All studied patients $(n=40)$} \\
\cline { 2 - 3 } staging & No. & $\%$ \\
\hline Stage I & 21 & $52.5 \%$ \\
Stage II & 8 & $20 \%$ \\
Stage III & 6 & $15 \%$ \\
Stage IV & 5 & $12.5 \%$ \\
\hline
\end{tabular}


Table $\mathbf{5}$ Type of treatment of all studied patients

\begin{tabular}{lll}
\hline Type of & \multicolumn{2}{l}{ All studied patients $(n=40)$} \\
\cline { 2 - 3 } treatment & No. & $\%$ \\
\hline RFA & 10 & $25 \%$ \\
MWA & 5 & $12.5 \%$ \\
TACE & 15 & $37.5 \%$ \\
Combined & 10 & $25 \%$ \\
\hline
\end{tabular}

Discovery ST PET-CT, General Electric Medical Systems, Milwaukee, WI, USA). All examinations were carried out using two integrated PET/CT scanners (Ingenuity TF 128; Philips Healthcare, Cleveland, $\mathrm{OH}, \mathrm{USA}$ ) $1 \mathrm{~h}$ after intravenous administration of 7-11 mCi of 18F-FDG corresponding to the patient's body weight. The CT scan component of the PET/CT examination included non-contrast CT acquisition of the liver, arterial phase CT of the

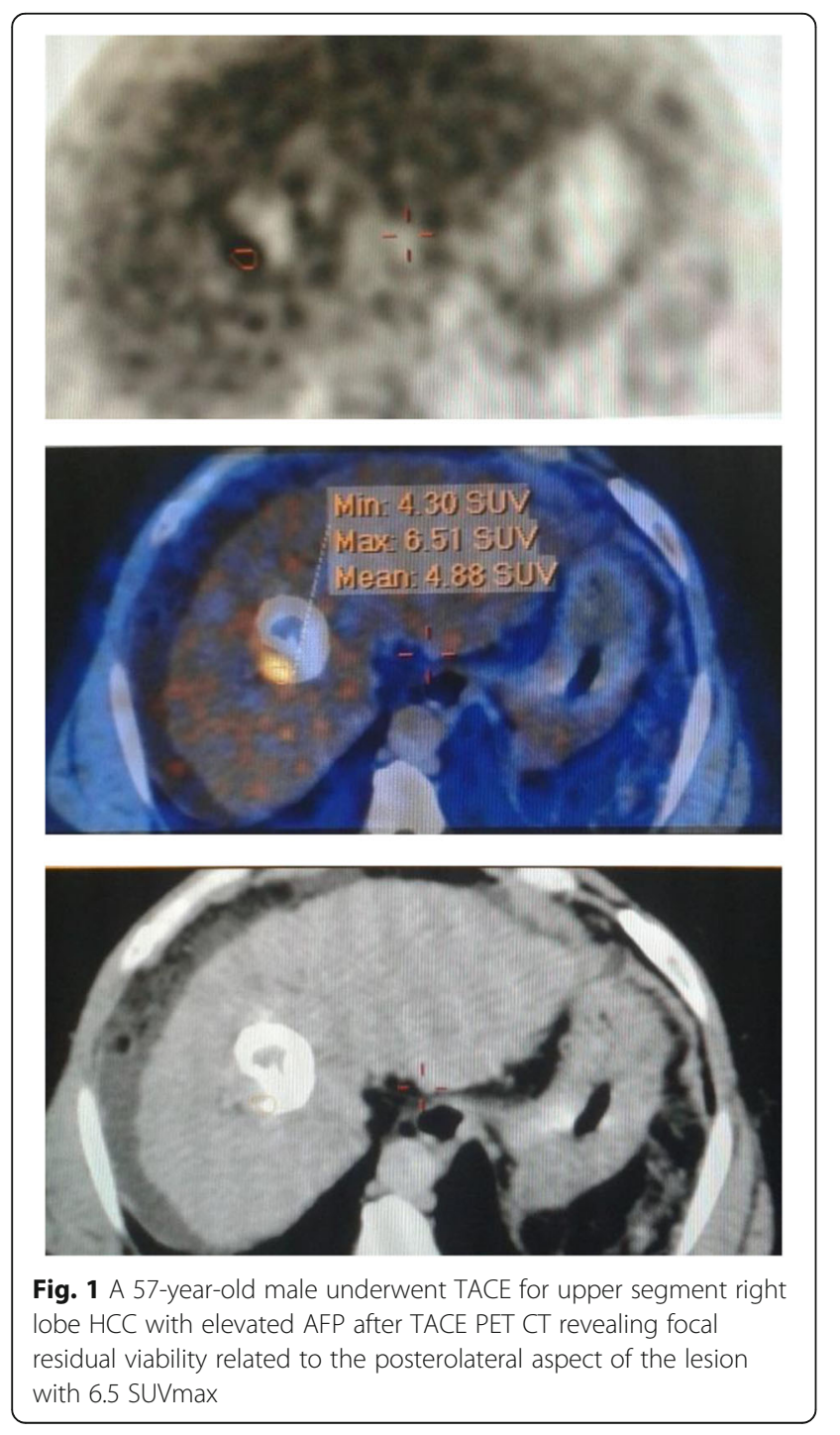

liver, portal venous whole-body CT, and equilibrium phase CT of the liver. For the arterial phase, the contrast agent iopromide (Ultravist) $(300 \mathrm{mg}$ of iodine $/ \mathrm{ml}$ ) was used at a dose of $100-120 \mathrm{ml}$ corresponding to the patient's body weight with a $3-\mathrm{ml} / \mathrm{s}$ infusion rate, following the administration of $50 \mathrm{ml}$ of a normal saline chaser at a $3-\mathrm{ml} / \mathrm{s}$ infusion rate. A 100-HU threshold was set in the region of interest (ROI) at the lower part of the descending thoracic aorta to trigger the start of hepatic arterial phase CT. The portal venous whole-body and equilibrium phases were acquired approximately 65 and $120 \mathrm{~s}$ after beginning the contrast medium infusion, respectively. During the portal venous phase, the patients were asked to breathe smoothly. The portal venous whole-body phase images were used for attenuation correction and integration with the PET images.

\section{Image analysis}

All CT images, attenuation-corrected PET images, and fused PET/CT images were transferred and viewed centrally on an interactive workstation (IntelliSpace Portal V4.0; Philips Healthcare). The 18FFDG PET images and contrast-enhanced CT (CECT) images were separated for interpretation (i.e., the 18F-FDG PET image findings were reviewed without knowledge of the CECT findings and vice versa). Two radiologists with 15 and 12 years of experience, respectively, in hepatic $\mathrm{CT}$ imaging reviewed all CECT components of the PET/CT scan. Two nuclear medicine physicians with 7 and 5 years of experience, respectively, reviewed all 18F-FDG PET images. All radiologists and nuclear medicine physicians were blinded to any clinical information or the results of the biopsy. Intrahepatic HCC recurrence was noted as newly developed lesions showing hyperenhancement in the arterial phase and washout in the delayed phase of the CECT component. In 18F-FDG PET/CT, disease activity was assessed either qualitatively or semi-quantitatively. Qualitative evaluation was based on the detection of focal 18F-FDG uptake that was higher than the surrounding background and distinct from tracer uptake physiological sites (e.g., bowel and myocardium), whereas semiquantitative evaluation typically relied on the calculation of the maximum standardized uptake value (SUVmax).

We calculated for each patient the SUVmax of the tumor and the ratio of the tumoral SUVmax to the normal liver SUVmax (TSUVmax/LSUVmax). In order to measure SUVmax for the tumor, we drew a $4 \times 4$ pixel square region of interest (ROI) and placed it on the area of the highest activity of the 
Table 6 PET/CT, biopsy results, and AFP values per response of all studied patients

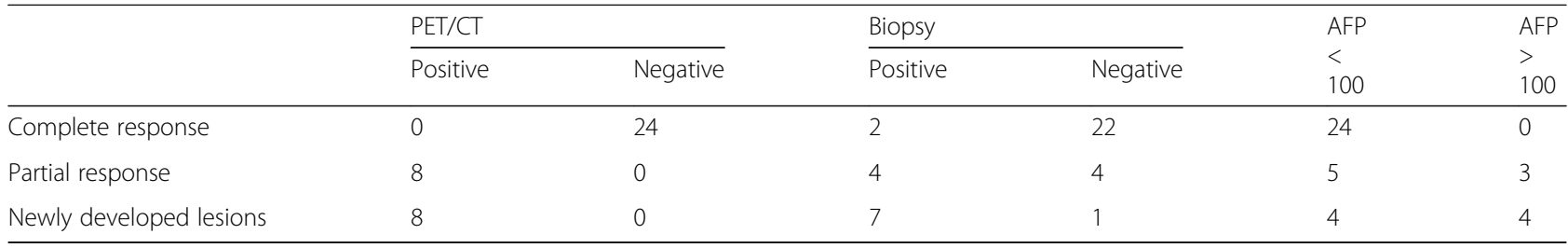

tumor but not covering the entire tumor, with the aid of combined CT and measured SUVmax in the ROI. In the case of multiple tumors, the SUVmax of the tumors was defined as the highest SUVmax of the tumors. To measure SUVmax for normal liver, we drew two 50-pixel circular ROIs and placed one on right lobe and one on liver transplantation (LT) lobe at a location where tumor was not detected on combined CT. The SUVmax of the normal liver was defined as the highest SUVmax of the two ROIs drawn on the normal liver. In this combined protocol, we established the diagnosis based on the combined findings from each modality. A finding was considered positive when it was observed in either the CECT or 18F-FDG PET/CT scan or both. We categorized intrahepatic HCC recurrence as either recurrence adjoining the treated site or at a site further than the original tumor site.

\section{Results}

The patients (Table 1 ) included 26 men and 14 women. The mean age of the enrolled patients was $60 \pm 11.7$ years (range, 25 - 82 years), and the mean duration of follow-up was 8 months (range, 1 - 59 months). Twenty-four (60\%) patients had hepatitis B virus (HBV) infection, six (15\%) patients had hepatitis C (HCV) infection, and ten (25\%) patients had alcoholic liver disease (Table 2).

The Child-Pugh classification (Table 3) was A in 18 (45\%) patients, B in $14(35.0 \%)$ patients, and C in $8(20$ $\%)$ patients.

According to the TNM staging system for HCC (Table 4), $21(52.5 \%)$ patients had stage I disease, 8 (20\%) had stage II disease, 6 (15\%) had stage III disease, and 5 (12.5\%) had stage IV disease.

Ten (25\%) patients underwent radiofrequency ablation (RFA), 5 (12.5\%) patients underwent microwave ablation (MWA), 13 (37.5\%) patients underwent transarterial
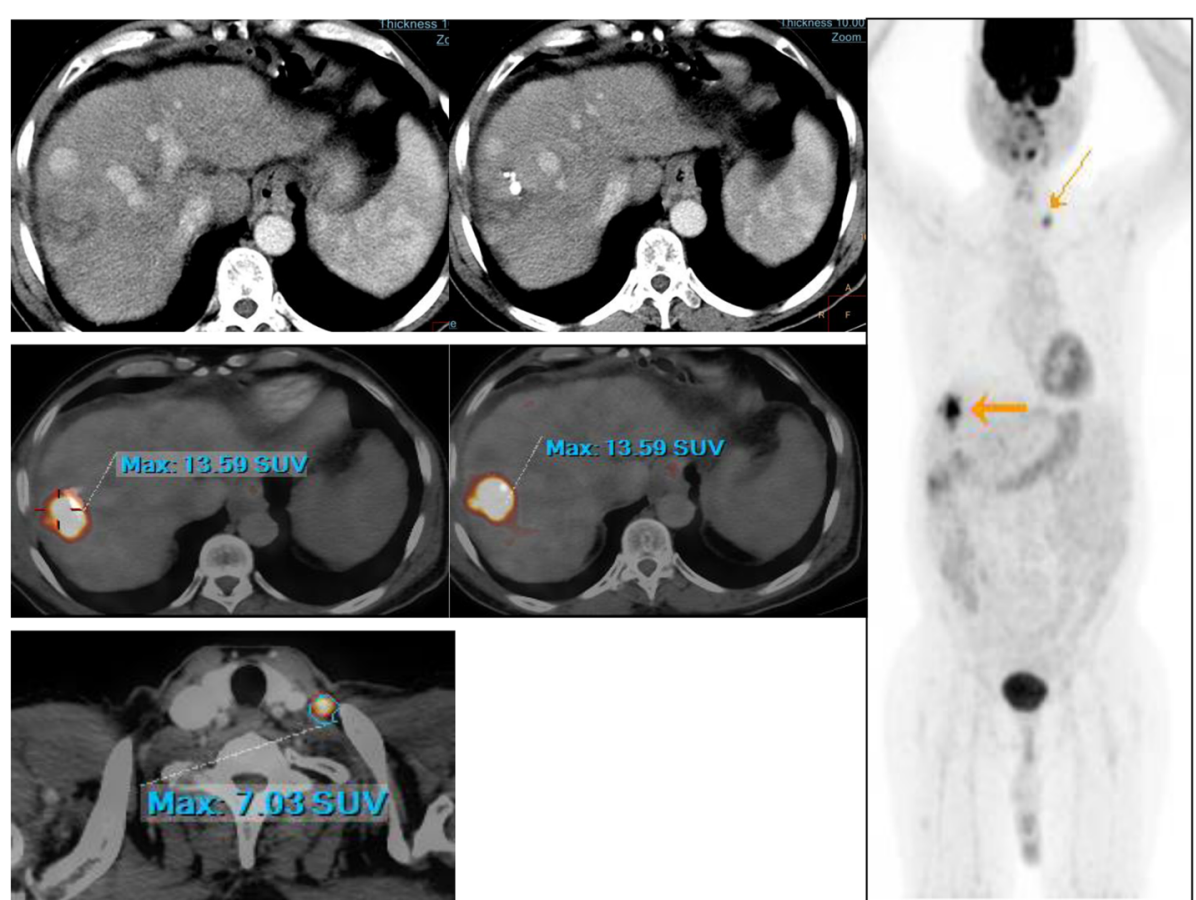

Fig. 2 A 63-year-old male having a history of HCC submitted for TACE 1.5 years ago currently presented with AFP 269. Diagnostic CT was negative, yet persistent rising of AFP PET revealed intense metabolic activity of the whole lesion achieving 13.5 SUVmax with metastatic left supra clavicular LN [7.03 SUVmax] 

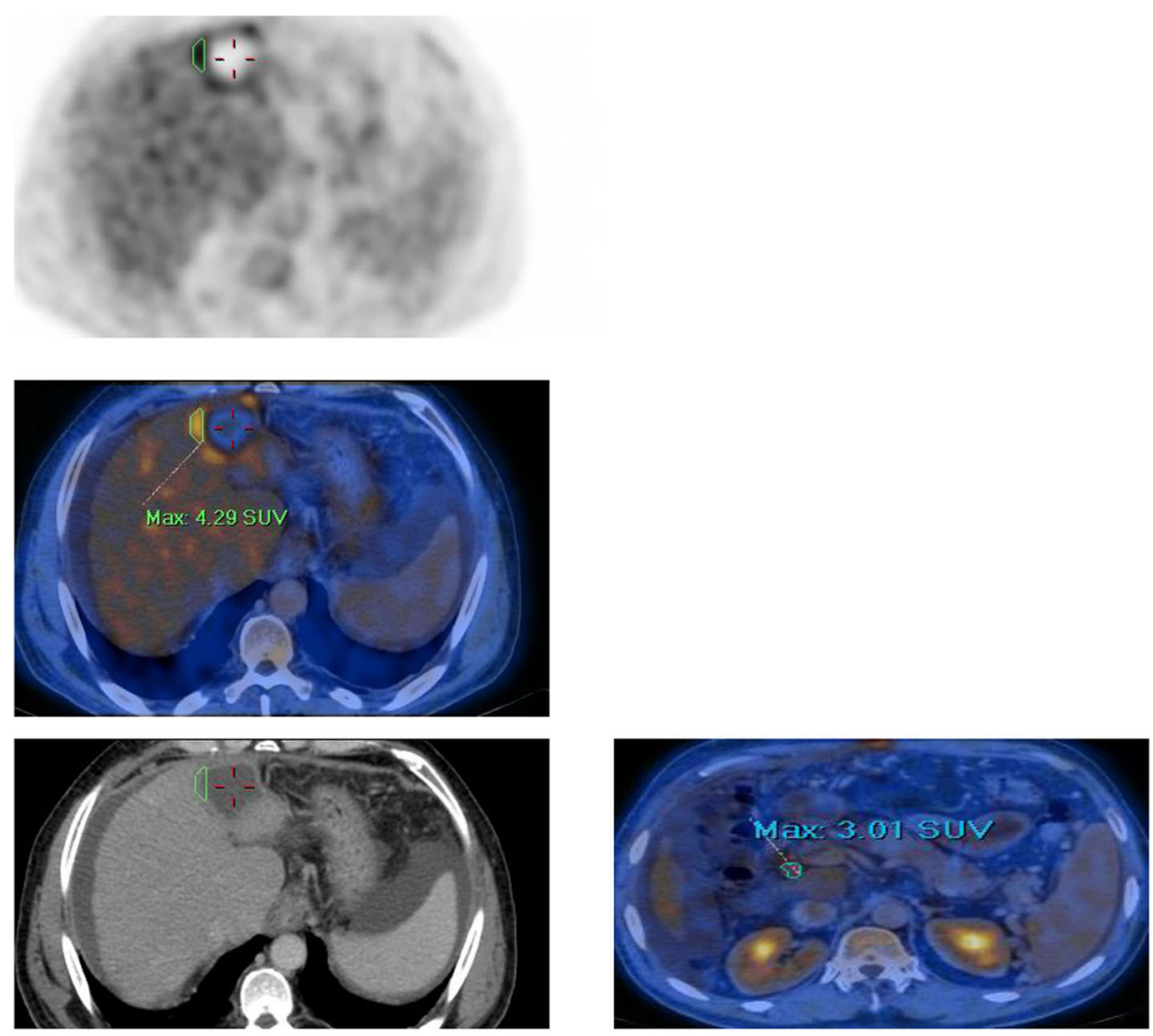

A

B

Fig. 3 A 50-year-old with HCC underwent RFA 2 months ago. The AFP was 4603 before the ablation and significantly dropped to 185 after RFA, then elevated again to 3500; PET CT is requested for further evaluation. a Clear detection of the marginal activity of the previously ablated lesion with SUVmax 4.2 along its lateral aspect. b Increased activity along a small mesenteric lymph node

chemoembolization (TACE), and 10 (25\%) patients received combined technique (Table 5).

Twenty-four patients were diagnosed as complete response after management and confirmed by PET/ CT. Eight patients showed focal residual/recurrence (Fig. 1), and eight patients showed newly developed lesions (Figs. 2, 3, 4 and 5). A biopsy (Table 6) was taken from the cases, in which biopsy was positive in 13 cases and negative in the other 27 cases. In 16 patients with positive FDG PET scan, alpha fetoprotein (AFP) level was less than $100 \mathrm{ng} / \mathrm{ml}$ in 9 patients and more than $100 \mathrm{ng} / \mathrm{ml}$ in 7 patients.

Regarding tumor size detected by FDG PET, HCC size was $>5 \mathrm{~cm}$ in eight cases and $<5 \mathrm{~cm}$ in eight

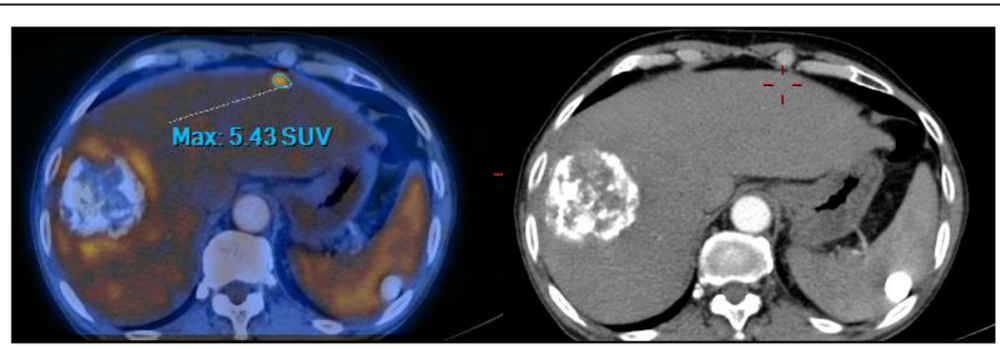

Fig. 4 A 67-year-old male patient, with right lobe hepatic focal lesion, underwent TACE 1 month ago presented now with elevated AFP referred for PET/CT assessment which revealed marginal increased FDG avidity that could represent residual activity or reactive changes, with another metabolically active left lobar subcapsular focus, mostly representing small newly developed HCC 


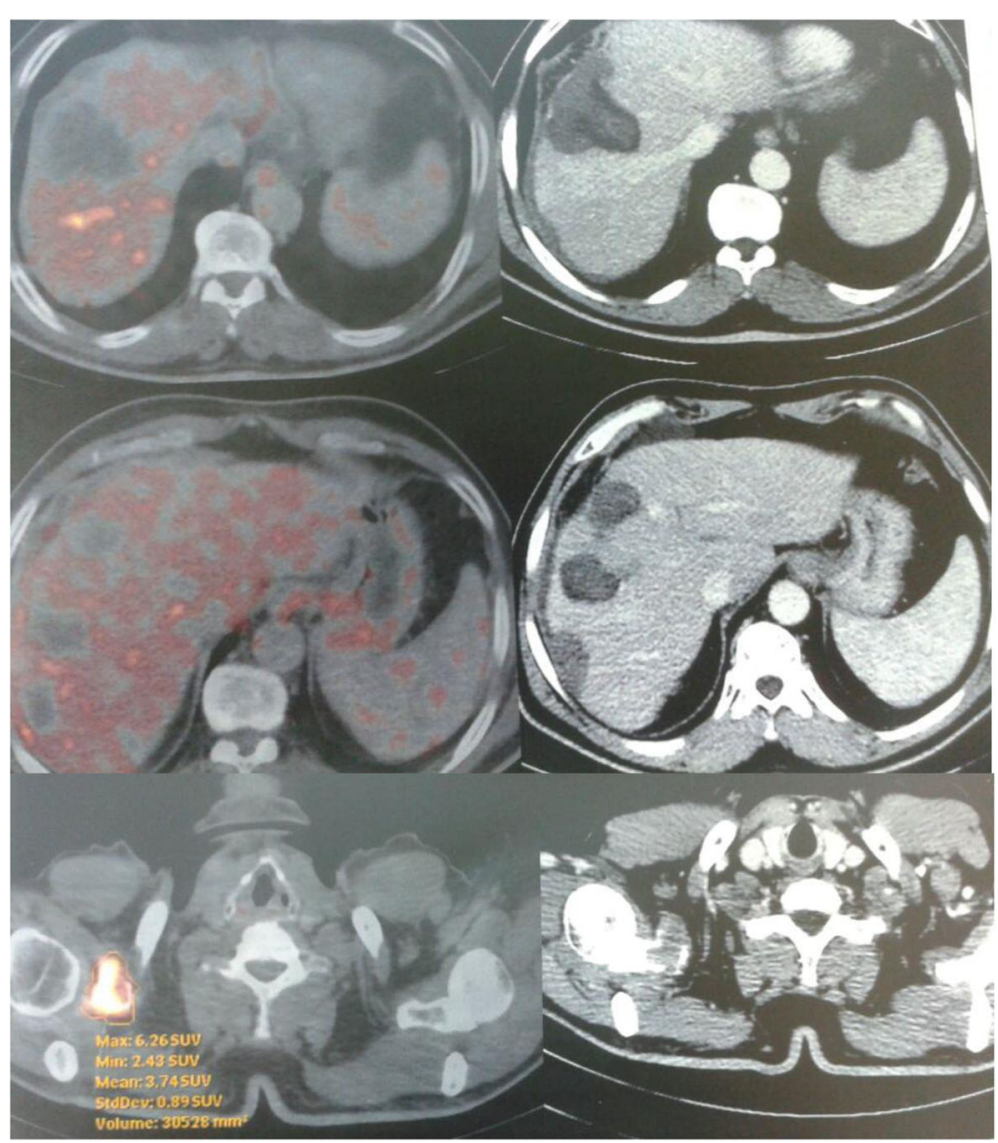

Fig. 5 A 50-year-old male patient with an elevated AFP level despite previous adequate RFA of three focal lesions. PET/CT revealed metastatic focal osteolytic lesion related to the right scapular glenoid notch achieving 6.2 SUVmax denoting metastatic nature

cases. Histologically, there were six well-differentiated, six moderately differentiated, and one poorly differentiated HCCs. FDG PET detected four of the six well- and all the six moderately differentiated HCCs plus the poorly differentiated HCCs. The intensity was evenly distributed between the different histologic grades. There was a strong correlation of FDG uptake with tumor size. In eight HCCs $>5 \mathrm{~cm}$ in size, four showed intense uptake on the scan. The other eight HCCs were $<$ or $=5 \mathrm{~cm}$ in size, and six were negative for uptake. The sensitivity of FDG PET in detecting $\mathrm{HCC}<$ or $=5 \mathrm{~cm}$ in size is low and therefore may not be helpful in detecting all of these tumors. For larger tumors, there is a strong correlation of sensitivity and uptake intensity with tumor size and elevated AFP levels. FDG PET sensitivity and uptake intensity did not correlate with histologic grade.

Hence, PET/CT achieved sensitivity $=92.8 \%$, specificity $=88.4 \%$, accuracy $=90 \%$, positive predictive value $=81.25$, and negative predictive value $=95.8$

\section{Discussion}

$\mathrm{HCC}$ is the commonest primary hepatic malignancy among adults. Globally, it is the fifth most common cancer and the third most common cause of cancer death [16]. In Egypt, HCC represents $11.75 \%$ of the malignancies of GIT and $1.68 \%$ of the total malignancies. HCC forms $70 \%$ of all hepatic tumors among Egyptians. Both hepatitis B virus (HBV) and hepatitis $\mathrm{C}$ virus $(\mathrm{HCV})$ represent a main risk factor for HCC in Egypt other than others causes, e.g., alcoholic liver disease [17].

FDG retention in malignant cells is dependent on intracellular glucose-6-phosphatase enzymatic activity [18]. Healthy liver cells contain high level of glucose-6-phosphatase and a small amount of hexokinase, but this ratio is reversed in the malignant HCC cells. This inconsistency enables FDG to accumulate in HCC but not in normal parenchymal cells [19]. HCCs contain varying levels of this enzyme and therefore reported sensitivity of FDG PET/CT scans in detecting hepatocellular carcinoma ranges between 50 and $70 \%$. Low sensitivity and variation in FDG uptake have been the main reasons for not routinely undergoing FDG PET/CT in HCC work up. Despite accuracy in diagnosing $\mathrm{HCC}, \mathrm{CT}$ and MRI cannot distinguish a well-differentiated $\mathrm{HCC}$ from a poorly 
differentiated HCC. Since most HCCs are not biopsied, FDG PET may play a role in predicting tumor characteristics and behavior non-invasively, as the variability of FDG uptake has been related to $\mathrm{HCC}$ differentiation and proliferative activity of HCC [20].

In the current study, we evaluated usefulness of FDG PET/CT in predicting prognosis of HCC. In which, we examined 40 known cases of HCC treated by different interventional techniques by using FDG PET/CT. The most important result in this study was that 24 cases showed complete metabolic response (CMR), 8 cases had partial metabolic response (PMR), and the other 8 cases showed newly developed lesions. A biopsy was taken from all cases in which 12 out of 40 were positive. The biopsy results were correlated with PET/CT findings, in which only two cases with CMR, four cases with PMR, and six cases with newly developed lesions had positive biopsies. So, in 40 patients with HCC, PET attained $83 \%$ sensitivity, $79 \%$ specificity, and $80 \%$ accuracy in the prediction of response.

Chen et al. [21] performed 31 FDG PET studies in 26 patients who had undergone either surgical resection or interventional therapy for HCC. During their follow up, they were noted to have high AFP serum levels. The sensitivity, specificity, accuracy, positive predictive value, and negative predictive value of FDG PET studies for detecting HCC recurrence were $73.3 \%, 100 \%, 74.2 \%, 100 \%$, and $11.1 \%$, respectively. Thirty patients were included in the study done by Omar et al. [22] in which all patients had history of local treatment of HCC; most of them were treated with TACE or RFA. They had undergone 18F-FDG $\mathrm{PET} / \mathrm{CT}$ for evaluation of the therapeutic effect after the end of the therapy which showed sensitivity of 18F-FDG PET/CT for detecting HCC recurrence was $96.5 \%$ while the specificity was about $83.3 \%$. Current study results agree with Refaat et al. [23] who studied $100 \mathrm{HCC}$ patients who were waiting for LT and who previously underwent locoregional therapy (LRT); they concluded that FDG PET/CT prediction of recurrence had sensitivity of $92.8 \%$ and a specificity of $94.1 \%$. Limitations of the current study were mainly a small number of the studied population.

\section{Conclusion}

Hepatocellular carcinoma (HCC) represents a common malignancy. PET/CT has an essential role in the follow-up of HCC patients after interventional management (e.g., TACE).

\section{Abbreviations}

AFP: Alpha fetoproteinCECTContrast-enhanced computed tomographyCMRComplete metabolic

responseFDGFluorodeoxyglucoseHBVHepatitis B virusHCCHepatocellular

carcinomaHCVHepatitis C virusLTLiver transplantationMRIMagnetic resonance
imagingMWAMicrowave ablationPET/CTPositron emission tomographycomputed tomographyPMRPartial metabolic responseRFARadiofrequency ablationROIRegion of interestSUVmaxMaximum standardized uptake valueTACETransarterial chemoembolizationTumor, Node, MetastasisStaging system for cancer

\section{Acknowledgements}

Not applicable

\section{Authors' contributions}

$\mathrm{AM}, \mathrm{MM}$, and OA conceived and designed the analysis. SR collected the data, performed the analysis, and wrote the manuscript. All authors read and approved the final manuscript.

\section{Funding}

The authors state that this work has not received any funding.

\section{Availability of data and materials}

The datasets used and/or analyzed during the current study are available from the corresponding author on reasonable request.

\section{Ethics approval and consent to participate}

This study was approved by the ethical committee of Ain Shams University on June 2016 (no reference number was given at that time). All patients included in this study gave written informed consent to participate in this research by the patients themselves or by primary degree relatives.

\section{Consent for publication}

Patients included in this research gave written informed consent to publish the data contained within this study.

\section{Competing interests}

The authors declare that they have no competing interests.

Received: 6 July 2019 Accepted: 26 February 2020

Published online: 06 April 2020

\section{References}

1. Janevska D, Chaloska-Ivanova V, Janevski V (2015) Hepatocellular carcinoma: risk factors, diagnosis and treatment. Open Access Maced J Med Sci. 3(4): $732-736$

2. Sanyal AJ, Yoon SK, Lencioni R (2010) The etiology of hepatocellular carcinoma and consequences for treatment. Oncologist. 15(suppl 4):14-22

3. Venook A, Papandreou C, Furuse J, Ladrón de Guevara L (2010). The incidence and epidemiology of hepatocellular carcinoma: a global and regional perspective the oncologist. 15:5-13.

4. Ferenci P, Fried M, Labrecque D, Bruix J, Sherman M, Omata M et al (2010) World Gastroenterology Organisation guideline. Hepatocellular carcinoma (HCC): a global perspective. J Gastrointestin Liver Dis 19:311-317.7

5. Guo J, Seo Y, Ren S (2016) Diagnostic performance of contrast-enhanced multidetector computed tomography and gadoxetic acid disodiumenhanced magnetic resonance imaging in detecting hepatocellular carcinoma: direct comparison and a meta-analysis. Abdom Radiol 41:1960

6. Choi JY, Lee JM, Sirlin CB (2014) CT and MR imaging diagnosis and staging of hepatocellular carcinoma: part II. Extracellular agents, hepatobiliary agents, and ancillary imaging features. Radiology 273(1):30-50

7. Di Martino M, Marin D, Guerrisi A et al (2010) Intraindividual comparison of gadoxetate disodium-enhanced MR imaging and 64-section multidetector $\mathrm{CT}$ in the detection of hepatocellular carcinoma in patients with cirrhosis. Radiology 256(3):806-816

8. Agrawal A, Rangarajan V (2015) Appropriateness criteria of FDG PET/CT in oncology. Indian J Radiol Imaging 25(2):88-101

9. Sharma B, Martin A, Zerizer I (2013) Positron emission tomographycomputed tomography in liver imaging. Semin Ultrasound CT MR 34:66-80

10. Liao X, Wei J, Li Y (2018) 18F-FDG PET with or without CT in the diagnosis of extrahepatic metastases or local residual/recurrent hepatocellular carcinoma. Medicine (Baltimore) 97(34):e11970

11. Lin CY, Chen JH, Liang JA et al (2011) 18F-FDG PET or PET/CT for detecting extrahepatic metastases or recurrent hepatocellular carcinoma: a systematic review and meta-analysis. Eur J Radiol 81:2417-2422 
12. Park JW, Kim JH, Kim SK (2008) A prospective evaluation of 18 F-FDG and $11 \mathrm{C}$-acetate PET/CT for detection of primary and metastatic hepatocellular carcinoma. J Nucl Med 49:1912-1921

13. Sun L, Guan YS, Pan WM et al (2009) Metabolic restaging of hepatocellular carcinoma using whole-body 18F-FDG PET/CT. World J Hepatol 1:90-97

14. Kuehl H, Stattaus J, Hertel $\mathrm{S}$ et al (2008) Mid-term outcome of positron emission tomography/computed tomography-assisted radiofrequency ablation in primary and secondary liver tumours - a single-centre experience. Clin Oncol (R Coll Radiol) 20:234-240

15. Kamel IR, Bluemke DA, Eng J et al (2006) The role of functional MR imaging in the assessment of tumor response after chemoembolization in patients with hepatocellular carcinoma. J Vasc Interv Radiol 17:505-512

16. Liao X, Wei J, Li Y et al (2018) 18F-FDG PET with or without CT in the diagnosis of extrahepatic metastases or local residual/recurrent hepatocellular carcinoma. Medicine 97:34

17. Holah NS, El-Azab DS, Aiad HA, Sweed DM (2015) Hepatocellular carcinoma in Egypt: epidemiological and histopathological properties. Menoufia Med J 28:718-724

18. Burt BM, Humm JL, Kooby DA et al (2001) Using positron emission tomography with [(18)F]FDG to predict tumor behavior in experimental colorectal cancer. Neoplasia 3(3):189-195

19. Cho KJ, Choi NK, Shin MH, Chong AR (2017) Clinical usefulness of FDG-PET in patients with hepatocellular carcinoma undergoing surgical resection. Ann Hepatobiliary Pancreat Surg 21(4):194-198

20. Abuodeh Y, Naghavi AO, Ahmed KA et al (2016) Prognostic value of pretreatment F-18-FDG PET-CT in patients with hepatocellular carcinoma undergoing radioembolization. World J Gastroenterol. 22(47):10406-10414

21. Chen Y, Hsieh D, Liao C, Bai C, Su C, Shen Y, Hsieh J, Liao A, Kao C (2005). Utility of FDG-PET for investigating unexplained serum AFP elevation in patients with suspected hepatocellular carcinoma recurrence. Anticancer Research vol. 25; 6C, 4719-4725.

22. Omar H, Mohamed E, Ayman M, Sherif S (2017) Role of PET/CT in assessment of post therapeutic hepatocellular carcinoma. Egypt I Hospital Med 69(4):2271-2277

23. Refaat R, Basha M, Hassan M (2018) Efficacy of contrast-enhanced FDG PET/ $\mathrm{CT}$ in patients awaiting liver transplantation with rising alpha-fetoprotein after bridge therapy of hepatocellular carcinoma. Eur Radiol 28:5356

\section{Publisher's Note}

Springer Nature remains neutral with regard to jurisdictional claims in published maps and institutional affiliations.

\section{Submit your manuscript to a SpringerOpen ${ }^{\circ}$ journal and benefit from:}

- Convenient online submission

- Rigorous peer review

- Open access: articles freely available online

- High visibility within the field

- Retaining the copyright to your article

Submit your next manuscript at $\boldsymbol{\nabla}$ springeropen.com 